\title{
Condorcet Consistency of Approval Voting: a counter example in Large Poisson Games *
}

\author{
Matías Núñez ${ }^{\dagger}$
}

May 2009

\begin{abstract}
Approval Voting is analyzed in a context of large elections with strategic voters: the Myerson's Large Poisson Games. We first establish the Magnitude Equivalence Theorem which substantially reduces the complexity of computing the magnitudes of the pivot outcomes. Furthermore, we show that the Condorcet Winner need not be the Winner of the election in equilibrium under Approval Voting. Indeed, a "paradoxical" example is provided where a candidate ranked first by more than half of the voters is not the Winner of the election.
\end{abstract}

KEYWORDS: Approval Voting, Poisson Games, Condorcet Winner.

JEL Classification Numbers: D72.

\section{Introduction}

The strategic analysis of voting rules has given some insight into the understanding of their properties. However, one can assert that these analyses are "too rich" in the sense that they show that a plethora of equilibria can arise under most voting rules. In particular, there is a controversy over Approval voting or $A V$, a voting rule which has been called "the electoral reform of the twentieth century". This voting rule allows the voter to vote for as many candidates as he wishes and the candidate who gets the most votes wins the election. Its detractors claim that this kind of method enhances strategic voting when compared for instance to Plurality voting, whereas its proponents consider that it has several advantages as far as strategic voting is concerned. For an extensive discussion on

\footnotetext{
*I wish to thank Jean-Frangis Laslier for his help and useful comments. I am also indebted to Francis Bloch, Steve Brams, Clémence Christin, Arnaud Dellis, Marc Leandri, François Maniquet, Jordi Massó, Vincent Merlin, Remzi Sanver, Giacomo Valletta and seminar and conference participants at Barcelona, Bilbao, Caen, Istambul, Lund, Montreal and Stony Brook for their valuable comments.

${ }^{\dagger}$ Département d'Economie, Ecole Polytechnique, 91128 Palaiseau, France. E-mail address: matias.nunez@polytechnique.edu
} 
this controversy over $A V$, the reader can refer to Brams [3], Brams and Fishburn [4], and Weber [14].

One important feature of $A V$ was characterized by Brams and Fishburn [2]. They show that if a Condorcet Winner exists then the $A V$ game has a Nash equilibrium in undominated strategies that selects the Condorcet Winner. The Condorcet Winner - the candidate who beats all other candidates on pairwise contests - has often been considered to be a good equilibrium solution in voting games. The robustness of this result has been weakened by De Sinopoli, Dutta and Laslier [5]. They apply refinements of Nash equilibrium such as the perfect equilibrium solution to Approval games. Using these techniques, they prove that in equilibrium these games can result in outcomes in which the Condorcet loser and Condorcet winner are selected with the same probability or even in which the Condorcet winner gets no vote at all. Therefore, $A V$ does not guarantee what is called Condorcet consistency: the Winner of the election does not always coincide with the Condorcet Winner.

However, the previous works were performed in a basic game theoretical framework. Such a framework faces some criticisms when dealing with elections with a large number of voters. Indeed, it is no longer realistic to assume that voters have no prior beliefs over the expected scores of the candidates. The existence of candidates with almost no chance of winning the election might affect voters' behavior as a voter might not vote for such a candidate. The introduction of such type of prior beliefs ${ }^{1}$ is the main objective of Large Elections models. To our knowledge, there exists two main models dealing with elections with a large number of strategic voters: the Score Uncertainty model (Laslier [9]) and the Population Uncertainty model (Myerson [10, 11, 12]).

The former is performed in a standard game theoretical framework where uncertainty is introduced by assuming that there is some small but strictly positive probability that each vote is wrongly recorded. Under this approach, Laslier shows that $A V$ leads to equilibria with desirable properties such as Condorcet Consistency and sincerity of voters' best responses. Myerson's Population Uncertainty framework, also known as Large Poisson Games $^{2}$, introduces an uncertainty over the total number of voters in the election. Indeed, it assumes that the total number of voters in the game is not constant and is drawn from a Poisson distribution of a given parameter $n$, the expected size of the population. Voters are assumed to be instrumentally motivated and consequently, their utility depends only on the candidate who wins the election. This implies that they only care about the influence

\footnotetext{
${ }^{1}$ A natural way of introducing this kind of prior beliefs is by means of Bayes-Nash equilibrium. However, as will be shown, the main advantage of Large Elections models when compared to this kind of solution concepts is that they provide a simpler way of working within these environments.

${ }^{2}$ Large Poisson Games are a novel field of research. Among the few works dealing with these games, the reader can refer to Myerson [10, 11, 12], Bouton and Castanheira [1], Goertz and Maniquet [7], Krishna and Morgan [8] and De Sinopoli and Gonzalez Pimienta [6].
} 
their ballot can have in pivotally changing the result of the election. Thus, the relative probabilities of pivot outcomes determine voters' best responses and hence the equilibria of the game.

Myerson [12] draws a positive conclusion over the properties of Approval voting when compared to other voting rules by analyzing some simple voting situations. However, one natural question that arises when confronted with this kind of models is: how important is this reduction in the set of equilibria in Large Poisson Games? In other words, does $A V$ lead to "desirable" equilibria in any voting situation?

In order to answer these questions, this paper focuses on Poisson voting games in which the expected number of voters $n$ tends towards infinity. In such situations, we use magnitudes of outcomes rather than probabilities of outcomes in order to compare their relative likelihood. The magnitude of an outcome measures the speed of convergence of the probability of this outcome towards zero as defined by Myerson [11, 12]. We first provide a result which simplifies the computation of the magnitudes of pivot outcomes: the Magnitude Equivalence Theorem $(M E T)$. This theorem provides a simple way to compute the magnitudes of this type of outcomes through a simple constrained minimization problem.

Besides, we also prove through a simple voting situation that Condorcet Consistency is not generically satisfied. Indeed, we provide an example where a candidate who is ranked first by more than half of the population (and thus the Condorcet Winner) is not the Winner of the election in equilibrium. In equilibrium, voters anticipate that the Condorcet Winner is not included in the most probable pivot outcome. This information concerning the probability of affecting the outcome of the election makes the majority of the voters vote for their preferred and for their second preferred candidate and this leads to the election of the latter. The existence of such an equilibrium is a consequence of the correlation between the scores of the candidates that arise under Approval Voting in Large Poisson Games. This example shows that the refinement of the set of Nash equilibria on Large Poisson Games is limited. However, in the described situation, there exists other equilibria in which other the Condorcet Winner wins the election.

This paper is structured as follows. Section 2 introduces the basic model. Section 3, presents the Magnitude Equivalence Theorem (MET) and Section 4 discusses in detail the situation where the Condorcet Winner does not coincide with the Winner of the election. Section 5 concludes. 


\section{The model}

\subsection{The basic setting}

A Poisson random variable $\mathcal{P}(n)$ is a discrete probability distribution that depends on a unique parameter which represents its mean. The probability that a Poisson random variable of parameter $n$ takes the value $l$, being $l$ a nonnegative integer is equal to

$$
e^{-n} \frac{n^{l}}{l !}
$$

A Poisson voting Game of expected size $n$ is a game such that the actual number of voters taking part in the election is a random variable drawn from a Poisson distribution with mean $n$. This assumption represents the uncertainty faced by voters w.r.t. the number of voters that show up the day of the election. The probability distribution and its parameter $n$ are common knowledge.

Each voter has a type $t$ that determines his preferences over the set of candidates $K=\{k, l, \ldots\}$. The preferences of a voter with a type $t$ is denoted by $u_{t}=\left(u_{t}(k)\right)_{k \in K}$. Thus, for a given $t, u_{t}(j)>u_{t}(k)$ implies that $t$-voters strictly prefer candidate $j$ to candidate $k$. Each type $t$ belongs to the set of types $T$.

Each voter's type is independently drawn from $T$ according to the distribution of types denoted by $r=(r(t))_{t \in T}{ }^{3}$. In other words, $r(t)$ represents the probability that a voter randomly drawn from the population has type $t$.

A finite Poisson game of expected size $n$ is then represented by $(K, T, n, r, u)$. A large Poisson game simply represents the limit when $n$ tends towards infinity of a finite Poisson game of expected size $n$.

For any pair of candidates $k, j \in K$, let $T_{k, j}=\left\{t \in T \mid u_{t}(k)>u_{t}(j)\right\}$ be the set of preference types where candidate $k$ is strictly preferred to candidate $j$. The Condorcet Winner (C.W.) of the election is defined as:

Definition 1. A candidate $k$ is called the Condorcet Winner (C.W.) of the election if

$$
\sum_{t \in T_{k, j}} r(t)>1 / 2 \forall j \in K, j \neq k \text {. }
$$

In order to completely determine an election in a Poisson voting game, the voting rule remains to be specified. Each voter $i$ must choose a ballot $c$ from a finite set of possible ballots denoted by $C$. Then, a Poisson Approval voting game will be represented by $(K, T, C, n, r, u)$ in which $C \subset K$ as an $A V$ ballot simply consists of a subset of the set of candidates.

\footnotetext{
${ }^{3}$ The distribution of types satisfies $r(t)>0 \forall t \in T$ and $\sum_{t \in T} r(t)=1$.
} 
As shown by Myerson [10], assuming a Poisson population has two main advantages: common public information and independence of actions.

As usual, voters' actions depend on their type (private information) and on the actions of other voters. In such a probabilistic framework, there exists a probability distribution over the different possible outcomes that might arise in the election. When we refer to common public information, we mean that this probability distribution does not depend on the type $t$. Indeed, each voter in the election fully knows the probability distribution over the different outcomes independently of $t$. This is not the case when using solution concepts such as the perfect equilibrium of Selten [13]. In a perfect equilibrium, strategic voters have some prior beliefs over the expected scores of the candidates. However, in such an equilibrium, there is an asymmetry of information that makes more difficult the analysis of the game. This common public information property of Poisson Games entails that voters' actions uniquely depend on their private information $t$ on this type of games in equilibrium.

The second main advantage is usually referred as the independence of actions. Indeed, the number of voters who choose a given ballot is independent from the number of voters who choose another ballot. This is not the case if we assume for instance a binomial distribution. Let us assume that a binomial random variable represents the number of voters in the election. A binomial distribution is characterized by two parameters $n$ and $p$. Whereas $p$ represents the probability of taking part in the election, the parameter $n$ stands for the maximal size of the population. This upper-bound for the number of voters implies that voters' actions are correlated. Indeed, if two voters vote for a given candidate $a$, there is at most $n-2$ voters that can vote for other candidates. This is not the case in a Poisson voting game as there is not an upper-bound for the number of voters in the election. These two properties substantially simplify the analysis of the voting game and are unique to the Poisson games as shown by Myerson [10].

We represent voters' actions by the strategy function $\sigma(c \mid t)^{4}$ which is a function from $T$ into $\Delta(C)$ the set of probability distributions over $C$. Formally, we will write

$$
\begin{gathered}
\sigma: T \longrightarrow \Delta(C) \\
t \longmapsto \sigma(. \mid t) .
\end{gathered}
$$

A voter with type $t$ chooses ballot $c$ with probability $\sigma(c \mid t)$. Then, taking into account the distribution of types $r$ and the strategy function $\sigma(. \mid t)$, the vote distribution $\tau=(\tau(c))_{c \in C}$ can be determined as follows. For each $c \in C$, we define

\footnotetext{
${ }^{4}$ The strategy function satisfies $\sigma(c \mid t) \geq 0 \forall c \in C$ and $\sum_{d \in C} \sigma(d \mid t)=1$.
} 


$$
\tau(c)=\sum_{t \in T} r(t) \sigma(c \mid t)
$$

The vote distribution $\tau$ represents the share of votes each ballot gets. We denote by $x(c)$ the Poisson random variable with parameter $n \tau(c)$ that describes the number of voters $x(c)$ who choose ballot $c$. Furthermore the vote profile $x=(x(c))_{c \in C}$ is a vector of length $C$ of independent random variables (due to the independent actions property).

The set of electoral outcomes ${ }^{5}$ given ballot set $C$ is denoted by $B(C)$, where

$$
B(C)=\left\{b \in \mathbb{R}^{C} \mid b(c) \text { is a non-negative integer for all } c \in C\right\}
$$

We denote by $b \in B(C)$ a vector of length $C$ of non-negative integer numbers. Each component $b(c)$ of vector $b$ accounts for the number of voters who vote for ballot $c$. The subsets of $B(C)$ will be denoted by capital letters $B \in B(C)$.

Given the vote profile $x$, the (common knowledge) probability that the outcome is equal to a vector $b \in B(C)$ is such that

$$
\begin{aligned}
P[x=b \mid n \tau] & =P\left[\bigcap_{c \in C} x(c)=b(c) \mid n \tau\right] \\
& =\prod_{c \in C} P[x(c)=b(c) \mid n \tau] \\
& =\prod_{c \in C}\left(\frac{e^{-n \tau(c)}(n \tau(c))^{b(c)}}{b(c) !}\right) .
\end{aligned}
$$

For ease of notation, we refer to $P[x=b \mid n \tau]$ by $P[x=b]$. We will be mainly interested in computing the probabilities of subsets of $B(C)$ rather than probabilities of vectors themselves, as for instance the probability of two given ballots getting the same number of votes. Given the vote profile $x$, we write that the probability of the outcome $B \in B(C)$ is equal to

$$
P[x=B]=\sum_{b \in B} P[x=b] .
$$

Let $\mathcal{C}_{k}$ denote the set of ballots in which candidate $k$ is approved. Given the vote profile $x$, the score distribution $\rho=(\rho(k))_{k \in K}$ describes the share of votes that each candidate gets. For each $k \in K$,

$$
\rho(k)=\sum_{c \in \mathcal{C}_{k}} \tau(c) .
$$

\footnotetext{
${ }^{5}$ In probabilistic terminology, an electoral outcome is usually referred as an event or realization of a random variable, i.e. the value that is actually observed (what actually happened). For ease of notation, we will refer to them simply as outcomes.
} 
It follows that the number of voters that vote for a candidate $k$ is drawn from a Poisson random variable with mean $n \rho(k)$. Given the score distribution, we define the score profile $s=(s(k))_{k \in K}$ describes the number of voters who vote for each candidate $k$ with

$$
s(k)=\sum_{c \in \mathcal{C}_{k}} x(c) \sim \mathcal{P}(n \rho(k))
$$

Given that under $A V$ voters can vote for several candidates, it is not generically true that the score profile $s$ is a vector of independent random variables. As will be shown this is an important property of $A V$ on Poisson games. Indeed, due to this correlation between the candidate scores, counterintuitive situations might arise.

Given an outcome $B \in B(C)$, let $M(B)=\arg \max _{j \in K} \rho(j)$ denote the set of candidates with the most points. Assuming a fair toss of a coin, the probability of candidate $k$ winning the election given the vector $B \in B(C)$ is

$$
Q[k \mid B]= \begin{cases}1 / \#(M(B)) & \text { if } k \in M(B) \\ 0 & \text { if } k \notin M(B) .\end{cases}
$$

For any outcome $B \in B(C)$ and any ballot $c \in C$, we let $B+\{c\}$ denote the outcome such that one ballot $c$ is added. That is, we write that the outcome $D \in B(C)$ is such that

$$
D=B+\{c\}=\{d \in D \subset B(C) \mid d(c)=b(c)+c(c) \text { for any } b \in B \subset B(C), c \in C\} .
$$

Thus, given the vote profile $x$, a voter with type $t$ casts the ballot $c$ that maximizes his expected utility

$$
E[c \mid n \tau]=\sum_{B \in B(C)} P[x=B] \sum_{k \in K} Q[k \mid B+\{c\}] u_{t}(k)
$$

We refer to $\{\sigma, \tau\}$ as an equilibrium of the finite Poisson voting game $(K, T, C, n, r, u)$ if $\tau$ is the vote distribution corresponding to the strategy function $\sigma$ and, for each $c \in C$ and each $t \in T$,

$$
\sigma(c \mid t)>0 \Longrightarrow c \in \arg \max _{d \in C} E[d \mid n \tau] .
$$

Nevertheless, as the focus of this work is on elections with a large number of voters, one shall look at the limits of equilibria as the expected number of voters $n$ tends to infinity. Thus, we refer to a large equilibrium sequence of $(K, T, C, r, u)$ to denote any equilibria sequence $\left\{\left(\sigma_{n}, \tau_{n}\right)\right\}_{n \rightarrow \infty}$ of the finite voting games $(K, T, C, n, r, u)$ such that the vectors $\left(\sigma_{n}, \tau_{n}\right)$ are convergent to some limit $(\sigma, \tau)$ as $n \rightarrow \infty$ in the sequence. We refer to this 
limit $(\sigma, \tau)$ as a large equilibrium of $(K, T, C, r, u)$. Furthermore, we refer to a sequence of outcomes in $B(C)$ by $\left\{B_{n}\right\}_{n \rightarrow \infty}$. The limit $B$ of a sequence of outcomes $\left\{B_{n}\right\}_{n \rightarrow \infty}$ in $B(C)$ is an outcome and so it is a subset of $B(C)$.

\subsection{Strategic behavior of the voters.}

As previously stated, we assume that each voter determines which ballot he casts by maximizing his expected utility. As voters are instrumentally motivated, they care only about the influence of their own vote in determining the Winner's identity. As usual in voting environments with a large number of voters, a voter's action has a negligible impact on the outcome of the election. Indeed, it has some impact only if there is some set of candidates involved in a close race for first place where one ballot could pivotally change the result of the election: a pivot.

Definition 2. Given the score profile $x$ and a subset $Y$ of the set of candidates $K$, an outcome $B \in B(C)$ is a pivot $(Y)$ if and only if:

$$
\begin{aligned}
& \forall y \in Y, \quad s(y) \geq \max _{k \in K} s(k)-1 \\
& \forall k \notin Y, \quad s(k)<\max _{k \in K} s(k)-2 .
\end{aligned}
$$

The set of all pivot outcomes is denoted by $\Sigma(C) \subset B(C)$, where

$$
\Sigma(C)=\{B \in B(C) \mid \exists Y \subset K, B=\operatorname{pivot}(Y)\}
$$

Besides, the set of all pivot outcomes in which candidate $k$ is involved is denoted by $\Sigma(C, k) \subset \Sigma(C)$, where

$$
\Sigma(C, k)=\{B \in \Sigma(C) \mid \exists Y \subset K \text { s.t. } k \in Y \text { and } B=\operatorname{pivot}(Y)\} .
$$

Thus, given the vote profile $\tau$, the expected utility for a voter with type $t$ of casting ballot $c$ is such that

$$
\begin{aligned}
E[c \mid n \tau] & =\sum_{B \in B(C)} P[x=B] \sum_{k \in K} Q[k \mid x+\{c\}] u_{t}(k) \\
& =\sum_{B \in \Sigma(C)} P[x=B] \sum_{k \in K} Q[k \mid x+\{c\}] u_{t}(k) .
\end{aligned}
$$

The probability of any pivot outcome generally tends to zero as the expected population $n$ becomes large. However, we can still compare their likelihood by comparing the rates at which their probabilities tend to zero. These rates can be measured by a concept of 
magnitude, defined as follows.

Given a large equilibrium sequence $\left\{\sigma_{n}, \tau_{n}\right\}_{n \rightarrow \infty}$, the magnitude $\mu[B]$ of an outcome $B \in B(C)$ is such that

$$
\mu[B]=\lim _{n \rightarrow \infty} \frac{1}{n} \log P[x=B \mid n \tau]=\lim _{n \rightarrow \infty} \frac{1}{n} \log P[x=B] .
$$

Notice that the magnitude of an outcome must be inferior or equal to zero, since the logarithm of a probability is never positive. The main advantage of using magnitudes is to have an analytical way to compare likelihoods of outcomes rather than estimations.

If one can show that a pivot between one pair of candidates has a magnitude that is strictly greater than the magnitude of a pivot between another pair of candidates, then the latter becomes infinitely less likely as the expected number of voters goes to infinity. That is to say, given two subsets $Y$ and $Y^{\prime}$ of the set of candidates $K$, for any pair of outcomes $\operatorname{pivot}(Y)$ and $\operatorname{pivot}\left(Y^{\prime}\right) \in B(C)$, if

$$
\mu[\operatorname{pivot}(Y)]>\mu\left[\operatorname{pivot}\left(Y^{\prime}\right)\right],
$$

then we know that the pivot outcome between candidates in $Y$ is infinitely more likely than the pivot outcome between candidates in $Y^{\prime}$, i.e.

$$
\lim _{n \rightarrow \infty} \frac{P\left[x=\operatorname{pivot}(Y)^{\prime}\right]}{P[x=\operatorname{pivot}(Y)]}=0
$$

\subsection{The Decision Process}

We now move to the description of the decision process of voters. Let $k$ be a candidate. Let $c$ and $c^{\prime}$ be two ballots that only differ by an extra candidate $k: c^{\prime}=c \cup k$. In order to evaluate which of the ballots the type- $t$ voter casts, he computes the sign of the following expression

$$
\begin{aligned}
\Delta & =E\left[c^{\prime} \mid n \tau\right]-E[c \mid n \tau] \\
& =\sum_{B \in \Sigma(C)} P[x=B] \sum_{k \in K}\left[Q\left[k \mid x=B+\left\{c^{\prime}\right\}\right]-Q[k \mid x=B+\{c\}]\right] u_{t}(k)
\end{aligned}
$$

The sum $\Delta$ simply represents the effect of adding candidate $k$ to his ballot in his expected utility. However, adding this extra candidate to his ballot can only have an impact in the cases where this candidate is involved in a pivot. Therefore, $\Delta$ can be rewritten as follows:

$$
\sum_{B \in \Sigma(C, k)} P[x=B] \sum_{k \in K}\left[Q\left[k \mid x=B+\left\{c^{\prime}\right\}\right]-Q[k \mid x=B+\{c\}]\right] u_{t}(k)
$$


Then, if there exists a $\operatorname{pivot}(Y) \in \Sigma(C, k)$ where candidate $k$ is involved which probability becomes infinitely more likely as $n$ tends towards infinity than every other pivot $b \in \Sigma(C, k)$, one can factor out by this pivot. Indeed, let us assume that every pivot $B$ where candidate $k$ is involved becomes infinitely less likely than $\operatorname{pivot}(Y)$ as the expected number of voters $n$ tends towards infinity, .

$$
\lim _{n \rightarrow \infty} \frac{P[x=B]}{P[x=\operatorname{pivot}(Y)]}=0 \quad \text { for all } B \in \Sigma(C, k)
$$

Given this focalisation of voters' attention on the outcome pivot $(Y)$, a voter's decision (the sign of $\Delta$ ) is reduced to evaluating which ballot maximizes his expected utility in case of a $\operatorname{pivot}(Y)$,

$$
\operatorname{sign}(\Delta)=\operatorname{sign}\left(\sum_{k \in K}\left[Q\left[k \mid x=\operatorname{pivot}(Y)+\left\{c^{\prime}\right\}\right]-Q[k \mid x=\operatorname{pivot}(Y)+\{c\}]\right] u_{t}(k)\right) .
$$

Repeating the previous procedure, one can deduce the best response for every voter in the election. Therefore, if given the vote profile $x$, there exists a strict ordering of the magnitudes of the pivot outcomes, we can ensure the existence of a unique best response.

\section{Computing magnitudes}

Two seminal papers $([11,12])$ state the main results that could be considered as the state-of-the-art techniques in the characterization of magnitudes in this type of games. The magnitude theorem sets up a method to compute such a limit as the solution of a maximization problem with a concave and smooth objective function. The dual magnitude theorem $(D M T)$ gives a method to compute magnitudes of outcomes that can be defined by linear inequalities involving the vote profile $x=(x(c))_{c \in C}$. The latter is now presented as it is necessary to introduce our main technical results. We first give the definition of offset ratio of an outcome that will be necessary throughout.

For any outcome $B \in B(C)$ and any ballot $c \in C$, the ratio $B(c) / n \tau_{n}(c)$ is called the $c$-offset ratio of $b$ when $n \tau_{n}$ is the vote distribution. That is, the $c$-offset is a ratio which describes the number of players who vote for ballot $c$ as a fraction of the expected number of voters who were supposed to cast ballot $c$.

For any ballot $c \in C$, we say that $\alpha(c)$ is the limit of $c$-offsets ${ }^{6}$ in the sequence of

\footnotetext{
${ }^{6}$ Technically speaking, $\alpha(c)$ is the limit of the major c-offsets. A sequence $\left\{b_{n}\right\}_{n \rightarrow \infty}$ is a major sequence of points in the sequence of outcomes $\left\{B_{n}\right\}_{n \rightarrow \infty}$ iff each $b_{n}$ is a point in $B_{n}$ and the sequence of points $\left\{b_{n}\right\}_{n \rightarrow \infty}$ has a magnitude that is equal to the greatest magnitude of any sequence that can be selected from the outcomes $B_{n}$. Formally, $b_{n} \in B_{n} \forall n$ and $\lim _{n \rightarrow \infty} \log \left(P\left[b_{n} \mid n \tau_{n}\right]\right) / n=$ $\lim _{n \rightarrow \infty} \max _{y \in B_{n}} \log \left(P\left[y \mid n \tau_{n}\right]\right) / n$. See Section 3 in Myerson [11] for a more detailed account of sequences of outcomes in Large Poisson Games.
} 
outcomes $\left\{B_{n}\right\}_{n \rightarrow \infty}$ iff $\left\{B_{n}\right\}_{n \rightarrow \infty}$ has a finite magnitude and, for every major sequence of points $\left\{b_{n}\right\}$ in $\left\{B_{n}\right\}_{n \rightarrow \infty}$, we have

$$
\alpha(c)=\lim _{n \rightarrow \infty} \frac{B_{n}(c)}{n \tau_{n}(c)}=\frac{B(c)}{n \tau(c)} \text { with } \tau(c)=\lim _{n \rightarrow \infty} \tau_{n}(c) \text { and } B(c)=\lim _{n \rightarrow \infty} \tau_{n}(c) .
$$

Theorem 1 (Dual Magnitude Theorem, Myerson [12]). Given the vote profile $x$, let $B \in$ $B(C)$ be an outcome defined by

$$
B=\left\{\sum_{c \in C} a_{k}(c) x(c) \geq 0 \forall k \in J\right\}
$$

in which $J$ is a finite set and parameters $a_{k}(c)$ are given for every $k \in J$ and $c \in C$. Suppose that $\lambda \in \mathbb{R}^{C}$ is an optimal solution to the problem

$$
\min _{\lambda} \sum_{c \in C} \tau(c)\left(\exp \left(\sum_{k} \lambda_{k} a_{k}(c)\right)-1\right) \quad \text { s.t. } \lambda_{k} \geq 0, \forall k \in J
$$

Then the optimal value of the objective function $(F)$ coincides with the magnitude $\mu[B]$ of the outcome $B \in B(C)$ and the limits of the c-offset ratios associated are such that

$$
\alpha(c)=\exp \left(\sum_{k} \lambda_{k} a_{k}(c)\right), \text { for all } c \in C
$$

This theorem states a simple technique to compute magnitudes of outcomes are defined w.r.t. to a finite series of inequalities.

As stated by Myerson [11], "the magnitude concept is useless to work" with outcomes "that differ by a single translation". Indeed, to compare the limit probabilities of this kind of outcomes, we need to use the offset theorem.

Let $w \in \mathbb{R}^{C}$ be a vector such that each component is a integer and $B \in B(C)$ an outcome. We denote the translation by vector $w$ of the outcome $B$ by $B-\{w\} \in B(C)$ the outcome in which adding vector $w$ would yield to the outcome $B$ :

$$
B-\{w\}=\{b-w \mid b \in B \text { and } b-w \in B(C)\} .
$$

Theorem 2 (Offset theorem, Myerson [11]). Let $w \in \mathbb{R}^{C}$ be a vector such that each component is non-negative integer. For each action $c$ such that $w(c)>0$, suppose that $\lim _{n \rightarrow \infty} n \tau(c)=\infty$ and that there exists an $\alpha(c)$ which is the limit of the c-offsets in the sequence of outcomes $\left\{B_{n}\right\}_{n \rightarrow \infty}$. Then, denoting by $B \in B(C)$ the outcome such that 
$B=\lim _{n \rightarrow \infty} B_{n}$, it follows that

$$
\lim _{n \rightarrow \infty} \frac{P[x=B-\{w\}]}{P[x=B]}=\prod_{c \in C} \alpha_{B}(c) .
$$

\subsection{Magnitude Equivalence Theorem}

The Magnitude Equivalence Theorem $(M E T)$ is the main technical result of this paper which substantially reduces the computations of the magnitude of a pivot outcome: it allows us to use directly the $D M T$ to compute magnitudes of pivot outcomes.

The $D M T$ is conceived to compute the magnitude of outcomes defined by a series of inequalities involving the vote profile $x=(x(c))_{c \in C}$. Formally, using the $D M T$ we compute the magnitude of an outcome $B \in B(C)$ defined by

$$
B=\left\{\sum_{c \in C} a_{k}(c) x(c) \geq 0 \forall k \in J\right\}
$$

However, a pivot outcome does not have this geometrical structure, i.e. for some $Y \subset K$, an outcome $\operatorname{pivot}(Y)$ is defined by

$$
\begin{aligned}
& \forall y \in Y, \quad s(y) \geq \max _{k \in K} s(k)-1 \\
& \forall k \notin Y, \quad s(k) \leq \max _{k \in K} s(k)-2 .
\end{aligned}
$$

Given that the components $s(k)$ of the score profile $s$ are sums of the components $x(c)$ of the vote profile $x$, i.e. $s(k)=\sum_{c \in \mathcal{C}_{k}} x(c)$, we cannot express a pivot outcome only using linear inequalities involving $x$.

The $M E T$ shows that the magnitude of a pivot outcome coincides with the magnitude of an outcome than can be defined uniquely using this type of inequalities.

We first provide a Claim which will be useful throughout. No proof is provided at it is a simple consequence of the definition of magnitude.

Claim 1. Let $\left\{a_{n}\right\}_{n \rightarrow \infty},\left\{b_{n}\right\}_{n \rightarrow \infty}$ be two sequence of vectors in $B(C)$ with finite magnitude. Given a large equilibrium sequence $\left\{\sigma_{n}, \tau_{n}\right\}_{n \rightarrow \infty}$, if there exists an $\left\{\varepsilon_{n}\right\}_{n \rightarrow \infty}$ such that

$$
\frac{P\left[x=a_{n}\right]}{P\left[x=b_{n}\right]}=\varepsilon_{n}
$$

with

$$
\lim _{n \rightarrow \infty} \frac{\log \left[\varepsilon_{n}\right]}{n}=0 \text { and } \varepsilon_{n}>0 \forall n \in \mathbb{N},
$$

then,

$$
\mu\left[a_{n}\right]=\mu\left[b_{n}\right] .
$$


Furthermore, any two sequence of outcomes $\left\{A_{n}\right\}_{n \rightarrow \infty},\left\{B_{n}\right\}_{n \rightarrow \infty}$ in $B(C)$ that satisfy condition (1) are such that $\mu\left[A_{n}\right]=\mu\left[B_{n}\right]$.

Theorem 3 (Magnitude Equivalence Theorem). Let $Y$ be a subset of $K$ and pivot $(Y)$ be its associated pivot outcome. Given a large equilibrium sequence $\left\{\sigma_{n}, \tau_{n}\right\}_{n \rightarrow \infty}$, we can write

$$
\mu[\operatorname{pivot}(Y)]=\mu[D]
$$

for some outcome $D \in B(C)$ defined by

$$
D=\{s(k)=s(l) \forall k, l \in Y\} \cap\{s(k) \geq s(l) \forall k \in Y \text { and } l \in K \backslash Y\} .
$$

Proof. By definition, given the score profile $x$ and a subset $Y$ of the set of candidates $K$, an outcome $B \in B(C)$ is a $\operatorname{pivot}(Y)$ if and only if:

$$
\begin{aligned}
& \forall y \in Y, \quad s(y) \geq \max _{k \in K} s(k)-1 \\
& \forall k \notin Y, \quad s(k)<\max _{k \in K} s(k)-1 .
\end{aligned}
$$

Given its definition, we can also express $\operatorname{pivot}(Y)$ as disjoint union $\bigcup_{m \in M} B_{m}$ of outcomes in $B(C)$ for some set $M$. In any of these outcomes $B_{m} \in B(C)$ included in this union, adding one extra ballot could pivotally change the election from some candidate $y$ in the set $Y$ to some other candidate $y^{\prime}$ in the set $Y$. Formally, the probability of $\operatorname{pivot}(Y)$ is such that

$$
P[x=\operatorname{pivot}(Y)]=P\left[x=\bigcup_{m \in M} B_{m}\right]=\sum_{m \in M} P\left[x=B_{m}\right],
$$

as the union is disjoint. Furthermore, we can write that any of these outcomes $B_{m} \in B(C)$ are the result of a translation with respect to the outcome $D \in B(C)$ defined by

$$
D=\{s(k)=s(l) \forall k, l \in Y\} \cap\{s(k) \geq s(l) \forall k \in Y \text { and } l \in K \backslash Y\} .
$$

Let $w_{m} \in \mathbb{R}^{C}$ be the vector of translation such that each component is an integer. In set theoretical terms, the outcome $B_{m}$ can be written as

$$
B_{m}=D-\left\{w_{m}\right\}=\left\{d-w_{m} \mid d \in D \text { and } D-w_{m} \in B(C)\right\}
$$

Let us suppose first that every offset ratio $\alpha(c)$ is different from zero and finite, where $c$ represents a ballot such that $\tau(c)>0$. Provided that $w_{m}(c)>0$, the offset theorem 
entails that

$$
\begin{aligned}
\lim _{n \rightarrow \infty} \frac{P\left[x=B_{m}\right]}{P[x=D]} & =\lim _{n \rightarrow \infty} \frac{P\left[x=D-\left\{w_{m}\right\}\right]}{P[x=D]} \\
& =\prod_{c \in C} \alpha(c)^{w_{m}(c)},
\end{aligned}
$$

where $\alpha(c)$ represents the $c$-offset ratio at the outcome $D$. In this case, the condition (1) of Claim 1 is satisfied as

$$
\lim _{n \rightarrow \infty} \frac{\log \left[\prod_{c \in C} \alpha(c)^{w_{m}(c)}\right]}{n}=0
$$

Therefore, by Claim 1 both outcomes $B_{m}$ and $D$ have the same magnitude. Denoting for every $m \in M$,

$$
v_{m}=\prod_{c \in C} \alpha(c)^{w_{m}(c)}
$$

the magnitude of the outcome $\operatorname{pivot}(Y)$ is such that

$$
\begin{aligned}
\mu[\text { pivot }(Y)] & =\lim _{n \rightarrow \infty} \frac{1}{n} \log \sum_{m \in M} P\left[x=B_{m}\right] \\
& =\lim _{n \rightarrow \infty} \frac{1}{n} \log \left[\sum_{m \in M} v_{m} P[x=D]\right] \\
& =\lim _{n \rightarrow \infty} \frac{1}{n} \log \left[P[x=D] \sum_{m \in M} v_{m}\right] \\
& =\lim _{n \rightarrow \infty} \frac{1}{n} \log P[x=D]+\lim _{n \rightarrow \infty} \frac{1}{n} \log \sum_{m \in M} v_{m} \\
& =\lim _{n \rightarrow \infty} \frac{1}{n} \log P[x=D]=\mu[D] .
\end{aligned}
$$

which shows that both magnitudes coincide when every offset ratio is finite and nonnegative. Repeating similar arguments proves the same equality whenever there exists the vector of translation $w_{m}$ is such that there is some $c \in C$ for which $w_{m}(c) \leq 0$.

Let us now suppose that there exists an $\alpha(c)$ which is equal to zero whenever $\tau(c)>0 .{ }^{7}$ Given the vote profile $x$ and the outcome $D$, we know that the limit of the number of voters who were supposed to vote for ballot $c$ is infinitely lower than the expected number

\footnotetext{
${ }^{7}$ It should be noted that an offset ratio does not only describe the limit of the number of players who vote for ballot $c$ as a fraction of the expected number of voters who were supposed to cast ballot $c$. Indeed, as a consequence of the offset theorem, the offset ratio $\alpha(c)$ of outcome $B$ also represents the limit when $n \rightarrow \infty$ of $P[x=B-c] / P[B]$. Therefore, if there exists an offset ratio $\alpha(c)=0$, then there must exist an outcome $B_{m}=D-\left\{w_{m}\right\}$ that belongs to the outcome pivot $(Y)$ such that $\lim _{n \rightarrow \infty} P[x=D] / P\left[x=B_{m}\right]=0$. For example, as $\alpha(c)$ represents the effect of subtracting one ballot $c$ to the outcome $D$ (i.e. $w_{m}(c)=c$ ), it suffices to take, for instance, the translation of adding ballot $c$, i.e. taking a translation vector $w_{m}$ with $w_{m}(c)=-c$.
} 
of voters who were supposed to do so. Formally,

$$
\alpha(c)=0 \Longrightarrow \lim _{n \rightarrow \infty} \frac{D(c)}{n \tau(c)}=0
$$

Due to the offset theorem, any outcome $B_{m}=D-\left\{w_{m}\right\}$ included in the outcome $\operatorname{pivot}(Y)$ such that $w_{m}(c)>0$ becomes infinitely less likely than the outcome $D$ as $n$ tends towards infinity. Formally, for any outcome $B_{m} \subset \operatorname{pivot}(Y)$,

$$
\begin{aligned}
& B_{m}=D-\left\{w_{m}\right\} \text { with } w_{m}(c)>0 \text { and } \alpha(c)=0 \\
& \quad \Longrightarrow \lim _{n \rightarrow \infty} \frac{P\left[x=B_{m}\right]}{P[x=D]}=\lim _{n \rightarrow \infty} \frac{P\left[x=D-\left\{w_{m}\right\}\right]}{P[x=D]}=0 .
\end{aligned}
$$

The ratio of the probability of any outcome $B_{m}=D-\left\{w_{m}\right\}$ included in the $\operatorname{pivot}(Y)$ in which $w_{m}(c)=0$ and the probability of the outcome $D$ is constant as by assumption there is a unique offset ratio which is equal to zero. Formally, the offset theorem entails that for any outcome $B_{m} \subset \operatorname{pivot}(Y)$,

$$
\begin{gathered}
B_{m}=D-\left\{w_{m}\right\} \text { with } w_{m}(c)=0 \text { and } \alpha(d) \neq 0 \forall d \in C, d \neq c \\
\Longrightarrow \lim _{n \rightarrow \infty} \frac{P\left[x=B_{m}\right]}{P[x=D]}=\prod_{d \in C \backslash c} \alpha(d)^{w_{m}(d)}=v_{m},
\end{gathered}
$$

for some $v_{m}$.

Finally, any outcome $B_{m}=D-\left\{w_{m}\right\}$ included in the outcome $\operatorname{pivot}(Y)$ in which $w_{m}(c)<$ 0 becomes infinitely more likely than the outcome $D$ as $n$ tends towards infinity but the magnitude of both outcomes coincide. Formally, for any outcome $B_{m} \subset \operatorname{pivot}(Y)$,

$$
\begin{aligned}
B_{m}=D-\left\{w_{m}\right\} \text { with } w_{m}(c)<0 & \text { and } \alpha(c)=0 \\
\Longrightarrow \lim _{n \rightarrow \infty} \frac{P[x=D]}{P\left[x=B_{m}\right]} & =\lim _{n \rightarrow \infty} \frac{P[x=D]}{P\left[x=D-\left\{w_{m}\right\}\right]} \\
& =\lim _{n \rightarrow \infty} \frac{P\left[x=D+\left\{w_{m}\right\}\right]}{P[x=D]}=0 .
\end{aligned}
$$

Indeed, the probability of the outcome $D \in B(C)$ is such that 


$$
P[x=D]=\prod_{c \in C} e^{-n \tau(c)} \frac{n \tau(c)^{D(c)}}{D(c) !} .
$$

Furthermore, as $w_{m}(c)<0$, the ratio of these probabilities can be expressed as follows:

$$
\begin{aligned}
\frac{P\left[x=D+\left\{w_{m}\right\}\right]}{P[x=D]} & =\prod_{d \in C \backslash c} \alpha(d)^{w_{m}(d)} \frac{[n \tau(c)]^{D(c)+w_{m}(c)}}{[n \tau(c)]^{D(c)}} \frac{D(c) !}{\left(D(c)+w_{m}(c)\right) !} \\
& =\prod_{d \in C \backslash c} \alpha(d)^{w_{m}(d)} \frac{D(c)(D(c)-1) \ldots\left(D(c)+w_{m}(c)+1\right)}{[n \tau(c)]^{-w_{m}(c)}}
\end{aligned}
$$

The ratio of probabilities $P\left[x=D+\left\{w_{m}\right\} / P[x=D]\right.$ converges to 0 as $n$ tends towards zero as $\prod_{d \in C \backslash c} \alpha(d)^{w_{m}(d)}$ does not depend on $n$ and by definition the offset ratio $\alpha(c)=0$ which implies that $\lim _{n \rightarrow \infty} D(c) / n \tau(c)=0$. However, even if the probabilities of the outcomes $B_{m}$ and $D$ diverge, their ratio $P[x=D] / P\left[x=B_{m}\right]$ satisfies Condition (1) of Claim 1:

$$
\begin{aligned}
\lim _{n \rightarrow \infty} & \frac{\log \left(P[x=D] / P\left[x=B_{m}\right]\right)}{n}= \\
& \lim _{n \rightarrow \infty} \log \left(\frac{D(c)(D(c)-1) \ldots\left(D(c)+w_{m}(c)+1\right)}{[n \tau(c)]^{-w_{m}(c)}}\right) / n=0,
\end{aligned}
$$

as by definition $\lim _{n \rightarrow \infty} D(c) / n \tau(c)=0$. As stated by Claim 1, the magnitude of the outcomes $D$ and $B_{m}$ coincide.

Every outcome $B_{m}$ which is included in the outcome $\operatorname{pivot}(Y)$ is either infinitely less likely than outcome $D$ as $n \rightarrow \infty$ (the outcomes $B_{m}=D-\left\{w_{m}\right\}$ such that $w_{m}(c)>0$ ) or has the same magnitude (the outcomes $B_{m}=D-\left\{w_{m}\right\}$ such that $w_{m}(c) \leq 0$ ). We can conclude that the magnitude of the outcome $\operatorname{pivot}(Y)$ coincides with the magnitude of outcome $D$ :

$$
\mu[\operatorname{pivot}(Y)]=\mu[D]
$$

Similar reasonings to the ones previously stated still apply when there several ballots with nil offset ratios.

This result shows that there exists an outcome, defined by a series of inequalities depending on the vote profile $x$, which magnitude coincides with the magnitude of the pivot outcome. Indeed, the outcome $D$ defined by Theorem 3 can be written down as:

$$
D=\left\{\sum_{c \in C} a_{k}(c) x(c) \geq 0 \forall k \in J\right\},
$$


for some parameters $a_{k}$ as, by definition,

$$
s(k)=\sum_{c \in \mathcal{C}_{k}} x(c)
$$

Thus, one can directly the $D M T$ to compute the magnitude of pivot outcomes, solving a simple constrained maximization problem.

\section{Approval Voting does not satisfy Condorcet Consistency}

In this section, an example is provided where, in equilibrium, the Winner of the election does not coincide with the Condorcet Winner. Moreover, in this equilibrium a candidate which is preferred by more than half of the voters is not elected. The majority of voters $\left(t_{2}\right.$-voters $)$ would prefer to vote just for their preferred candidate, candidate $b$. However, they vote for their second preferred candidate $a$ to prevent candidate $c$ from winning the election, as the most probable pivot outcome in which candidate $a$ is involved is against candidate $c$. It is a pure coordination problem which the Poisson uncertainty does not remove. This equilibrium is characterized by a failure in preference aggregation: it is due to the correlation between the scores of the candidates that naturally arise in Large Poisson Games when a voting rule allows to vote for several candidates.

Let us consider a Large Poisson Approval voting game where there are three candidates $K=\{a, b, c\}$ and three different types $T=\left\{t_{1}, t_{2}, t_{3}\right\}$ such that:

$$
\begin{array}{|c|c|c|}
t_{1} & t_{2} & t_{3} \\
\hline a & b & c \\
b & a & a \\
c & c & b \\
\hline
\end{array}
$$

in which the utility of $t_{1}$-voters satisfies $u_{t_{1}}(a)>u_{t_{1}}(b)>u_{t_{1}}(c)$ and so on. This example does not lie on the utility levels but rather on the preference orderings. Be3ides, the distribution of types satisfies

$$
r\left(t_{1}\right)=0.1, \quad r\left(t_{2}\right)=0.6 \text { and } r\left(t_{3}\right)=0.3 \text {. }
$$

Given this distribution, candidate $b$ is the $C . W$. as

$$
\begin{aligned}
r\left(t_{2}\right) & >r\left(t_{1}\right)+r\left(t_{3}\right) \\
r\left(t_{1}\right)+r\left(t_{2}\right) & >r\left(t_{3}\right),
\end{aligned}
$$

Furthermore, candidate $b$ Is more than simply a Condorcet Winner. There is more than 
half of the voters that rank him first.

Proposition 1. On Large Poisson Games, a candidate who is ranked first by more than the expected half of voters need not be the Winner of the election under AV in equilib2ium.

Proof. We claim that there is a large equilibrium $\{\sigma, \tau\}$ of the game $(K, T, C, r, u)$ in which candidate $b$ is the Winner of the election. In this large equilibrium, the strategy function satisfies

$$
\sigma\left(a \mid t_{1}\right)=\sigma\left(a, b \mid t_{2}\right)=\sigma\left(c \mid t_{3}\right)=1
$$

and the vote distribution is such that

$$
\tau(a)=r\left(t_{1}\right), \quad \tau(a, b)=r\left(t_{2}\right), \quad \tau(c)=r\left(t_{3}\right) .
$$

Given the vote distribution, the vote profile $x=(x(c))_{c \in C}$ is the following vector

$$
x(a) \sim \mathcal{P}(0.1 n), \quad x(a, b) \sim \mathcal{P}(0.6 n) \text { and } x(c) \sim \mathcal{P}(0.3 n) .
$$

In such an equilibrium, the score distribution $\rho=(\rho(k))_{k \in K}$ is such that

$$
\rho(a)=\tau(a)+\tau(a, b)=0.7, \rho(b)=\tau(a, b)=0.6 \text { and } \rho(c)=0.3
$$

Given this score distribution, the Winner of the election is candidate $a$ which therefore implies that $A V$ is not Condorcet Consistent in Poisson Games. Finally, given the score distribution, the score profile $s=(s(k))_{k \in K}$ is such that

$$
s(a)=x(a)+x(a, b) \sim \mathcal{P}(0.7 n), \quad s(b)=x(b) \sim \mathcal{P}(0.6 n) \quad \text { and } s(c)=x(c) \sim \mathcal{P}(0.3 n)
$$

Let us now show why $\{\sigma, \tau\}$ is indeed a large equilibrium of this Poisson Approval voting game. The aim is to prove that the pair $\{\sigma, \tau\}$ induces a probability distribution over the set of pivot outcomes such that $\{\sigma, \tau\}$ still are best responses of the game. The solved minimization problems are included in the appendix. In this example, there are three possible pivot outcomes involving two candidates $\operatorname{pivot}(a, b), \operatorname{pivot}(a, c)$ and $\operatorname{pivot}(b, c)$ and one pivot outcome in which the three candidates are involved.

Given the pair $\{\sigma, \tau\}$, the $M E T$ implies that the magnitude of the outcome $\operatorname{pivot}(a, b)$ is equal to the magnitude of the outcome $\{s(a)=s(b) \geq s(c)\}$. Formally, we write

$$
\mu[\operatorname{pivot}(a, b)]=\mu[\{s(a)=s(b) \geq s(c)\}] .
$$


The outcome $\{s(a)=s(b) \geq s(c)\}$ can be defined as

$$
\{[x(a) \geq 0] \cap[-x(a) \geq 0] \cap[x(a)+x(a, b)-x(c) \geq 0]\} .
$$

According to the $D M T$, we know that the magnitude of $\operatorname{pivot}(a, b)$ is equal to the solution of the following optimization problem.

$$
\tau(a) \exp \left[\lambda_{1}-\lambda_{2}+\lambda_{3}\right]+\tau(a, b) \exp \left[\lambda_{3}\right]+\tau(c) \exp \left[-\lambda_{3}\right]-1,
$$

such that $\lambda_{i} \geq 0 \forall i$. Thus, the magnitude of this pivot outcome is such that

$$
\mu[\operatorname{pivot}(a, b)]=-0.1 \text {. }
$$

Similarly, combining the $M E T$ and the $D M T$, the magnitude of a pivot between candidates $a$ and $c$ is equal to

$$
\mu[\operatorname{pivot}(a, c)]=\mu[\{s(a)=s(c) \geq s(b)\}]=-0.0834849,
$$

and the magnitude of a pivot between candidates $b$ and $c$ is equal to

$$
\mu[\operatorname{pivot}(b, c)]=\mu[\{s(b)=s(c) \geq s(a)\}]=-0.151472 .
$$

Moreover, the magnitude of the pivot between candidates $a, b$ and $c$ is equal to the magnitude of the pivot between candidates $b$ and $c$, i.e.

$$
\mu[\operatorname{pivot}(a, r, c)]=-0.151472=\mu[\operatorname{pivot}(b, c)] .
$$

Therefore, the magnitudes of the pivot outcomes are ordered as follows:

$$
\mu[\operatorname{pivot}(a, c)]>\mu[\operatorname{pivot}(a, b)]>\mu[\operatorname{pivot}(b, c)]=\mu[\operatorname{pivot}(a, f, c) .
$$

This ordering is not "intuitive" as candidates $a$ and $b$ both have a higher expected score than candidate $c$ and therefore one would expect that the most probable pivot outcome will include both of them. This a consequence of the correlations between the scores of the candidates that arise in the Poisson Games.

Taking into account the ordering of the magnitudes, one can determine the ballot that each voter of a given type chooses. As previously argued, a voter votes for a candidate $k$ iff the pivot outcome with the highest magnitude involving candidate $k$ is against a less preferred candidate. In this case, the magnitudes of the pivot outcomes are strictly ordered so that voters' best responses immediately follow. Therefore, the strategy function 
satisfies

$$
\sigma\left(a \mid t_{1}\right)=\sigma\left(a, b \mid t_{2}\right)=\sigma\left(c \mid t_{3}\right)=1
$$

and the vote distribution is such that

$$
\tau(a)=r\left(t_{1}\right), \quad \tau(a, b)=r\left(t_{2}\right), \quad \tau(c)=r\left(t_{3}\right),
$$

showing that $\{\sigma, \tau\}$ is a large equilibrium of the game $(K, T, C, r, u)$.

On single-peaked preferences One cannot escape from this type of bad equilibria by artificially restraining voters' preferences. This example can be extended to a situation in which preferences are single-peaked. Let us we assume that there are four different types $T=\left\{t_{0}, t_{1}, t_{2}, t_{3}\right\}$ such that

\begin{tabular}{|c|c|c|c|}
$t_{0}$ & $t_{1}$ & $t_{2}$ & $t_{3}$ \\
\hline$a$ & $a$ & $b$ & $c$ \\
$c$ & $b$ & $a$ & $a$ \\
$b$ & $c$ & $c$ & $b$ \\
\hline
\end{tabular}

in which the distribution of types $r$ satisfies

$$
r\left(t_{0}\right)=\varepsilon, \quad r\left(t_{2}\right)=0.1-\varepsilon, r\left(t_{3}\right)=0.6 \text { and } r\left(t_{4}\right)=0.3 .
$$

for some small $\varepsilon>0$. With such a slight alteration, the large equilibrium in which candidate $a$ is the Winner of the election still exists and the preference profile satisfies single-peakedness.

The equilibrium is not unique It is important to emphasize that in this game there is another large equilibrium in which the C.W. coincides with the Winner of the election. In such a large equilibrium, the strategy function $\sigma(. \mid t)$ satisfies

$$
\sigma\left(a \mid t_{1}\right)=\sigma\left(b \mid t_{2}\right)=\sigma\left(a, c \mid t_{3}\right)=1
$$

and the vote distribution is such that

$$
\tau(a)=0.1, \quad \tau(b)=0.6, \quad \tau(a, c)=0.3 .
$$

In this alternative equilibrium, the Winner of the election is candidate $b$. Indeed, in such an equilibrium, the outcome $\operatorname{pivot}(a, b)$ becomes infinitely more probable than any other 
pivot outcome $B \in \Sigma(C)$ as $n$ tends towards infinity. Voters with type $t_{1}$ and $t_{2}$ vote for their preferred candidate and the $t_{3}$-voters vote for candidate $a$ to prevent candidate $b$ in the event of an outcome pivot $(a, b)$.

\section{Conclusion}

This work analyses the properties of $A V$ on Large Poisson Games, one of the main models of large elections. Large Poisson Games possess several advantages such as the independent actions or the environmental equivalence property that simplify the analysis of the voting equilibria. Using these games, Myerson [12] shows that $A V$ is more robust to information manipulation than other one-shot voting rules such as Plurality voting in some simple voting games. This work shows that $A V$ does not preclude paradoxical situations from arising as a consequence of the independent actions property. When the voting rule allows to vote for more than one candidate, the fact that the number of voters who cast a given ballot is independent of the number of voters who cast another one (independent actions property) naturally implies that the scores of the candidates are correlated. The main problem is that, because of this correlation, the Winner of the election does not always coincide with the Condorcet Winner. Whenever the voters anticipate that the Condorcet Winner is not included in the most probable pivot outcome, he need not be the Winner of the election in equilibrium. This fact limits the reduction of Nash equilibria that arises in Large Poisson Games.

The failure of preference aggregation under $A V$ described within this work arises because voters vote given the relative likelihood of the different pivot outcomes. These pivot outcomes depend on the scores of candidates and not on the number of voters who cast a given ballot. As the scores are correlated, this leads to paradoxical situations. Indeed, in the Score Uncertainty model (Laslier [9]) candidates' scores are independent random variables. With such an independence, $A V$ ensures that voters' best responses are sincere and the Condorcet Winner wins the election whenever it exists, provided that every candidate gets a strictly positive share of votes.

\section{References}

[1] L. Bouton and M. Castanheira. One person, many votes: Divided majority and information aggregation. mimeo, E.C.A.R.E.S., 2008.

[2] S. Brams. Approval voting, condorcet's principle and runoff elections. Public Choice, 36:89-114, 1981. 
[3] S. Brams. Mathematics and Democracy: Designing Better Voting and Fair-Division Procedures. Princeton, NJ: Princeton University Press., 2008.

[4] S. Brams and P.C. Fishburn. Approval Voting. Birkhauser, Boston, 1983-2007.

[5] F. De Sinopoli, B. Dutta, and J-F. Laslier. Approval voting: three examples. International Journal of Game Theory, 38:27-38, 2006.

[6] F. De Sinopoli and C. Gonzalez Pimienta. Undominated (and) perfect equilibria in poisson games. Games and Economic Behaviour, forthcoming.

[7] J. Goertz and F. Maniquet. On the informational efficiency of approval voting. mimeo, C.O.R.E., 2008.

[8] V. Krishna and J. Morgan. On the benefits of costly voting. mimeo, Penn State University, 2008.

[9] J.F. Laslier. The leader rule: A model of strategic approval voting in a large electorate. Journal of Theoretical Politics, 21:113-136, 2009.

[10] R. Myerson. Extended poisson games and the condorcet jury theorem. Games and Economic Behavior, 25:111-131, 1998.

[11] R. Myerson. Large poisson games. Journal of Economic Theory, 94:7-45, 2000.

[12] R. Myerson. Comparison of scoring rules in poisson voting games. Journal of Economic Theory, 103:219-251, 2002.

[13] R. Selten. A reexamination of the perfectness concept for equilibrium points in extensive games. International Journal of Game Theory, 4:25-55, 1975.

[14] R. Weber. Approval voting. Journal of Economic Perspectives, 9:39-49, 1995.

\section{Appendix}

This appendix provides the constrained minimization problems used to compute the magnitudes of the pivot outcomes in section 4 , in the large equilibrium in which the Condorcet Winner does not coincide with the Winner of the election.

\section{Magnitude of a pivot between candidates $a$ and $b$}

$$
\begin{aligned}
\mu[\{s(a)=s(b) \geq s(c)\}]=\min _{\lambda} \tau(a) & \exp \left[\lambda_{1}-\lambda_{2}+\lambda_{3}\right] \\
& +\tau(a, b) \exp \left[\lambda_{3}\right]+\tau(c) \exp \left[-\lambda_{3}\right]-1
\end{aligned}
$$


such that $\lambda_{i} \geq 0 \forall i$. The solution to this problem yields

$$
\mu[\{s(a)=s(b) \geq s(c)\}]=\mu[\{x(a)=0\}]=-r\left(t_{1}\right) \text { as } r\left(t_{2}\right)>r\left(t_{3}\right) .
$$

Magnitude of a pivot between candidates $a$ and $c$

$$
\begin{aligned}
\mu[\{s(a)=s(c) \geq s(b)\}]=\min _{\lambda} & \tau(a) \exp \left[\lambda_{1}-\lambda_{2}+\lambda_{3}\right] \\
& +\tau(a, b) \exp \left[\lambda_{1}-\lambda_{2}\right]+\tau(c) \exp \left[-\lambda_{1}+\lambda_{2}\right]-1,
\end{aligned}
$$

such that $\lambda_{i} \geq 0 \forall i$. Therefore,

$$
\begin{aligned}
\mu[\operatorname{pivot}(a, c)] & =\mu[\{s(a)=s(c) \geq s(b)\}] \\
& =-\left(\sqrt{r\left(t_{1}\right)+r\left(t_{2}\right)}-\sqrt{r\left(t_{3}\right)}\right)^{2}=\mu[x(a)+x(a, b)=x(c)] .
\end{aligned}
$$

Magnitude of a pivot between candidates $b$ and $c$

$$
\begin{aligned}
\mu[\{s(b)=s(c) \geq s(a)\}]=\min _{\lambda} \tau(a) & \exp \left[-\lambda_{3}\right] \\
& +\tau(a, b) \exp \left[\lambda_{1}-\lambda_{2}\right]+\tau(c) \exp \left[-\lambda_{1}+\lambda_{2}\right]-1
\end{aligned}
$$

such that $\lambda_{i} \geq 0 \forall i$. Therefore,

$$
\begin{aligned}
\mu[\operatorname{pivot}(b, c)]=\mu[\{s(b)=s(c) \geq s(a)\}] & =-r\left(t_{1}\right)-\left(\sqrt{r\left(t_{2}\right)}-\sqrt{r\left(t_{3}\right)}\right)^{2} \\
& =-r\left(t_{1}\right)+\mu[x(a, b)=x(c)]=\mu[\operatorname{pivot}(a, b, c)] .
\end{aligned}
$$

